\title{
Chaotic Synchronization in a Small Network of a Class of Power Systems via Contraction Analysis
}

\author{
G. Solís-Perales, E. Ruiz-Velázquez, and J. A. García-Rodríguez
}

Departamento de Electrónica, CUCEI, Universidad de Guadalajara, Avenida Revolución No. 1500, 44430 Guadalajara, JAL, Mexico

Correspondence should be addressed to G. Solís-Perales, gualberto.solis@cucei.udg.mx

Received 12 April 2012; Revised 7 June 2012; Accepted 8 June 2012

Academic Editor: Jun-Juh Yan

Copyright (c) 2012 G. Solís-Perales et al. This is an open access article distributed under the Creative Commons Attribution License, which permits unrestricted use, distribution, and reproduction in any medium, provided the original work is properly cited.

This paper presents a synchronization analysis of networks of a class of power systems using the contraction theory for nonlinear systems. This analysis is characterized by not being based on Lyapunov's stability theory, that is, it is not required to determine a Lyapunov candidate function. Moreover, from the contraction conditions, robustness of the synchronization can be obtained, in this sense, the analysis method is robust. The analysis consists in identifying or proposing a virtual or auxiliary system which is contracting in a region of the state space. It is intended that in this region the trajectories of the systems on the network converge to those of the virtual system and then obtain the synchronization of the systems in the network. The contribution consists in applying this nontraditional analysis to the problem of chaotic synchronization of a network of a class of power systems.

\section{Introduction}

A network is defined as the interconnection of objects through a coupling, and they could be found almost everywhere, from social to genomic networks. For instance, in biological systems, electronic circuits, social communities, spread of diseases, and neuronal systems, among others. Its study and analysis have been attractive for researchers in science and engineering due to the diversity of problems and applications where these structures appear [1-3]. These networks present many challenges, for example, the problem of dynamic networks, that is, the network structure is changing over time and the size of the network can be static or not. The problem of considering nonlinear couplings implies that the coupling force between nodes is described by a nonlinear relationship of states or it is time varying; the problem of synchronization of networks with nonidentical nodes means that systems in the nodes are strictly different [4]. One problem that has attracted a lot of attention is the synchronization between nodes. In this sense, synchronization has been studied using 
many techniques. The main assumption considered as a starting point is that there is a reference signal where the trajectories of the systems in the network converge. For example, in [5], global synchronization and asymptotic stability were demonstrated, where sufficient conditions were established on the basis of Lyapunov's stability. In [6], a scheme based on state observers has been reported, where a network of systems with the same model in the nodes was considered, and only one scalar signal was used as the output and one as an input, the synchronization was obtained by the Lyapunov stability theory and the linear matrix inequalities (LMI) technique. Network synchronization can also be found in modular structures [7], where connections between communities is poor, these networks are of great importance in social or biological networks, where synchronization is obtained using the approach of the master stability function which requires the Lyapunov exponents. The abovementioned contributions require two main aspects, the determination of a Lypunov function, which in many cases is difficult to obtain, and the determination of the transversal Lyapunov exponent through a numerical process. In this contribution, an alternative technique to determine synchronization of a class of complex networks of power systems is applied. Such an alternative technique is the contraction theory, which can be used to ensure synchronization between systems [8], for example, in [9-11], the application of contraction theory to synchronization of systems in the master-slave form is shown. In this work, the contraction theory is applied to synchronization of power systems but in a network form. That is, a control system is not used for achieving synchronization, this is achieved by the interconnection between systems, whereas in the case of the master-slave synchronization, a control action is applied to the slave system in order to achieve synchronization. The relevant part is that the convergence of the system trajectories can be analyzed differentially instead of using the Lyapunov stability theory. Recently, the contraction theory and the techniques of the master stability function were analyzed for the synchronization of complex networks, and several important observations were established [12], for example, in the use of contraction theory, it is not required to determine a Lyapunov function or the Lyapunov exponents, whereas in the case of the master stability function, the Lyapunov exponents are essential.

In this contribution, we apply the contraction theory to study and analyze the convergence of trajectories instead of analyzing stability of an equilibrium point. Contraction theory provides a simple way to test the convergence of the trajectories, for this purpose, a virtual or auxiliary system is required. The convergence is achieved when the trajectories of all systems are in a region of the state space called contraction region and stay in this region for all future time; furthermore, the convergence between them is exponential.

We apply this analysis technique to the problem of synchronization of a class of power systems. It is worth mentioning that the contraction analysis is relatively recent, so there is few results on its application to power systems and even less about synchronization of networks of generators. Thus, in this contribution, we analyze the synchronization of a network of a class of power systems using a simple model for the power generators. This type of system is particularly interesting since they might present a complex behavior even chaos; therefore, we are interested in achieving chaotic synchronization of the systems. This type of power systems are interconnected in a network form, such that, they can represent an entire network of generation and supply of electric energy. It should be mentioned that, in this contribution, it is considered that the model is given by a second-order system with two state variables given by the angular position and angular velocity of the generator which are the variables to be synchronized. Applying the contraction theory for synchronization of the generators, a contraction region can be determined, which may depend on the system parameters, the coupling force, and the connectivity degree; the connectivity degree of 
the node is the number of connections of that node. The importance of the results is that we can analyze the network synchrony in electrical generators, where the main problem is to achieve synchrony mainly in power, since the network must be in balance or in synchrony; moreover, despite the simplicity of the analysis, a robustness of the synchronization can be claimed. The robustness can be obtained provided the contraction conditions holds; thus, the synchronization is robust against certain parameter variations.

The paper is organized as follows: in Section 2 basic results about contraction theory are presented, Section 3 describes the network synchronization of power systems, and, finally, Section 4 presents some conclusions about network synchronization of power systems.

\section{Basics on Contraction Theory}

The contraction theory permits to analyze stability of nonlinear systems in terms of convergence of the system trajectories using a differential approximation. This theory is different from the Lyapunov stability methods, specially because the knowledge of an equilibrium point is not necessary, therefore, the stability analysis is based on virtual displacements. The contractive behavior is a property regarding to the convergence of the trajectories of arbitrary systems; therefore, a nonlinear system is contracting if the effect of initial conditions is forgotten exponentially. Another important aspect of the contraction theory is that the differential stability analysis is accurate and it does not depend on a numerical calculation. Consider nonlinear systems of the form

$$
\dot{x}=F(x, t),
$$

where $x \in \mathbb{R}^{n}$ is the state vector, $F: \mathbb{R}^{n} \rightarrow \mathbb{R}^{n}$ is a nonlinear vector field continuously differentiable. Let $\delta x$ be an infinitesimal virtual displacement of the state $x$ at a fixed time; therefore, the first system variation is given by

$$
\delta \dot{x}=\frac{\partial F(x, t)}{\partial x} \delta x
$$

where the square of the distance $\left(\delta x^{T} \delta x\right)$ between adjacent trajectories is as follows:

$$
\begin{aligned}
\frac{d}{d t}\left(\delta x^{T} \delta x\right) & =2 \delta x^{T} \delta \dot{x}=2 \delta x^{T} \frac{\partial F}{\partial x} \delta x \\
& \leq 2 \lambda_{\max }(x, t) \delta x^{T} \delta x
\end{aligned}
$$

and therefore,

$$
\|\delta x\| \leq\left\|\delta x_{0}\right\| e^{\int_{0}^{t} \lambda_{\max }(x, t) d t} .
$$

Where $\lambda_{\max }(x, t)$ is the largest eigenvalue of the symmetric part of the Jacobian $J=(\partial F / \partial x)$, then, any infinitesimal displacement $\|\delta x\|$ converges to zero exponentially, that is, $\exists \beta>0$ for all $x, y$ for all $t \geq 0$, such that, $\lambda_{\max }(x, t) \leq-\beta<0$. Then, from (2.4) it is ensured that all solutions of the system in (2.1) converge exponentially to a single trajectory despite the effect of initial conditions. 
Definition 2.1. Given the system (2.1), a region C of the state space is called contraction region if the Jacobian matrix $J=(\partial F / \partial x)$ is uniformly negative definite in that region.

Therefore, the contraction principle can be stated as follows.

Theorem 2.2. Let $x(t)$ and $\tilde{x}(t)$ be two generic trajectories of the system (2.1). Let $\mathbf{M}_{t}: \mathbf{B}_{\varepsilon} x(t)$ be the tube around $x(t)$, and let $\mathbf{C} \in \mathbb{R}^{n}$ be a contraction region of phase space defined as

$$
C:=\left\{x \in \mathbb{R}^{n}:\left(\frac{1}{2}\right)\left(\left(\frac{\partial F}{\partial x}\right)+\left(\frac{\partial F}{\partial x}\right)^{T}\right) \leq-\beta I, \beta>0, \forall t \in \mathbb{R}^{+}\right\}
$$

If $\tilde{x}(t)$ is such that $\tilde{x}\left(x_{0}\right) \in \mathbf{M}_{0}$ and $\mathbf{M}_{t} \subset \mathbf{C}$, for all $t \in \mathbb{R}^{+}$then $\tilde{x}(t) \in \mathbf{M}_{t}$, for all $t \in \mathbb{R}^{+}$, and $\delta x^{T} \delta \dot{x}=k \delta x_{0}^{T} \delta x_{0} e^{-\beta t}$, with $k \geq 1, \beta \geq 0$, for all $t \in \mathbb{R}^{+}$.

This theorem states that given $\dot{x}=F(x, t)$, a trajectory that starts in a tube of constant radius centered on a given trajectory and contained in the region of contraction will remain in that ball and it converges to the given trajectory for all time. In the case where the entire state space is a region of contraction, the convergence of the trajectories is global. The fact that the Jacobian matrix is uniformly negative definite implies that

$$
\exists \beta>0, \quad \forall x, y \forall t \geq 0,
$$

such that

$$
\frac{1}{2}\left(\frac{\partial F}{\partial x}+\frac{\partial F^{T}}{\partial x}\right) \leq-\beta I<0
$$

Generalization of the convergence analysis using coordinate transformations $\delta z=\Theta(x, t) x$ with $\Theta(x, t)$ a uniform and invertible matrix can be found in [13-15].

\section{Synchronization of a Network of a Class of Power Systems}

A network of $N$ identical systems is described by the following system of equations:

$$
\dot{x}_{i}=F\left(x_{i}\right)+k \sum_{j=1}^{N} a_{i, j} \Gamma x_{j}
$$

for $i=1,2, \ldots, N$, where $x_{i}$ is the state vector of the $i$ th system or network node, $F$ : $\mathbb{R}^{n} \rightarrow \mathbb{R}^{n}$ is a smooth vector field representing the dynamics of the systems at the nodes, $\Gamma=\operatorname{diag}\left[\gamma_{1}, \gamma_{2}, \ldots, \gamma_{n}\right]$ is the input matrix that determines which states in the nodes are connected, and the $a_{i, j}$ are the elements of the $A$ matrix which describes the connectivity and network topology; it is considered that the connection between nodes is constant and also 
diffusive (the sum of the elements of the rows is zero), $k$ is the coupling strength between nodes

$$
\sum_{j=1}^{N} a_{i, j}=0, \quad a_{i, i}=-\sum_{\substack{j=1 \\ j \neq i}}^{N} a_{i, j} .
$$

In this contribution, the strongest form of synchronization between systems on a network is considered, that is, complete and global synchronization. This synchronization is defined in the invariant manifold $\mathcal{M}=\left\{x_{1}(t)=x_{2}(t)=\cdots=x_{N}(t)\right\}$. The dimension of this synchronization manifold is given by the dimension of only one system.

Definition 3.1. The network (3.1) is fully synchronized if

$$
\lim _{t \rightarrow \infty}\left\|x_{i}(t)-x_{j}(t)\right\|=0 \quad \forall i, j
$$

Now suppose the following to carry out the idea of synchronization of a power system network.

Assumption 3.2. The models of the generators in the network are identical and have all the same parameters but different initial conditions.

Assumption 3.3. The connectivity in the network is bidirectional, there is no isolated systems, and the network is static.

Assumption 3.2 is common in the study of complex network synchronization and it facilitates the determination of a virtual system for synchronization analysis; however, some parameter variations can be considered as we will see later. Assumption 3.3 states that if a system at certain node is connected to another, the latter also must be connected to the first one; that is, they are connected bidirectionally or mutually. Now, for the application of contraction results to the synchronization problem we have the following corollary.

Corollary 3.4. Suppose we have a network of $N$ systems described by (3.1), then a virtual system for the network is given by

$$
\phi\left(x, x_{i}\right)=F(x)+k a_{i, i} \Gamma x+k \sum_{\substack{j=1 \\ j \neq i}}^{N} a_{i, j} \Gamma x_{j},
$$

where the system (3.1) is a particular solution of (3.4) for any $i$. If the system is contracting respect to the variable $x$, then the systems on the network are synchronized.

Remark 3.5. Regarding robustness of the synchronization, it can be considered that system (3.4) is the nominal plant and all the particular solutions given by the systems in the network can present some parameter variations and the contraction region is then robust.

Now, consider the classical model of an electrical generator connected to an infinite bus [16-18] for a power system which is used for energy generation which is perturbed 


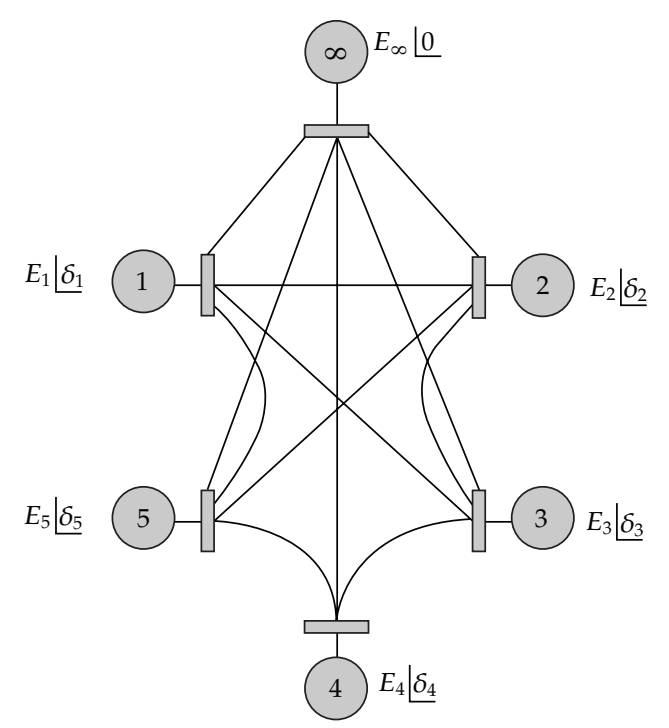

Figure 1: Network of power systems as generators connected to an infinite bus.

by a periodic signal. This is the simplest model used to identify and understand instability problems

$$
M \frac{d^{2} \delta}{d t^{2}}+D \frac{d \delta}{d t}+P_{\max } \sin (\delta)=P_{m}
$$

where $M$ is the moment of inertia, $D$ corresponds to the damping constant, $P_{\max }$ is the maximum power of the generator, $P_{m}=l \sin (\omega t)$ is the power of the machine, and $\delta$ represents the angular position. From this equation it is possible to rewrite the system as

$$
\begin{aligned}
& \dot{x}_{1}=x_{2}, \\
& \dot{x}_{2}=-c x_{2}-\beta \sin \left(x_{1}\right)+h \sin (\omega t),
\end{aligned}
$$

where we define the states $x_{1}=\delta, x_{2}=d \delta / d t$, and the positive parameters $c=D / M$, $\beta=P_{\max } / M, h=l / M$. These systems are considered to be connected in a network of electrical generation, where the objective is to present a synchronous behavior between them. It should be mentioned that these models exhibit a chaotic behavior for certain parameter values and due to the oscillatory input signal. It is considered that we have an interconnected network of five generators as in Figure 1, where the systems connected to the infinite bus are shown, $E_{i}$ and $\delta_{i}$ are voltage and the angular position in the $i$ th generator, respectively. To establish synchronization of the network using the contraction theory, a virtual system is proposed which has as a particular solution each of the systems in the network, this system is chosen as

$$
\dot{x}=f(x)+k a_{i, i} \Gamma x+k \sum_{\substack{j=1 \\ j \neq i}}^{N} a_{i, j} \Gamma x_{j} .
$$


It is important to mention that we chose the value $a_{i, i}$, since this value satisfies the particular solution for the $i$ th system; for example, if the network is globally connected, this value is simply $-N+1$; therefore, in general $a_{i, i}=-\operatorname{deg}\left\{x_{i}\right\}$, then, the virtual system with $\Gamma=\operatorname{diag}[1,0]$ for the power system, takes the form

$$
\begin{aligned}
& \dot{x}_{1}=x_{2}+k a_{i, i} x_{1}+k \sum_{\substack{j=1 \\
j \neq i}}^{N} a_{i, j} x_{1, j}, \\
& \dot{x}_{2}=-c x_{2}-\beta \sin \left(x_{1}\right)+h \sin (\omega t) .
\end{aligned}
$$

From Definition 2.1 the next Jacobian matrix is found as

$$
A=\frac{\partial \psi}{\partial x}=\left[\begin{array}{cc}
k a_{i, i} & 1 \\
-\beta \cos \left(x_{1}\right) & -c
\end{array}\right]
$$

Now, the negative symmetric part is calculated

$$
-\Lambda=-\frac{1}{2}\left(A+A^{T}\right)=\left[\begin{array}{cc}
-k a_{i, i} & -\frac{1}{2}+\frac{\beta}{2} \cos \left(x_{1}\right) \\
-\frac{1}{2}+\frac{\beta}{2} \cos \left(x_{1}\right) & c
\end{array}\right] .
$$

Then, to determine the convergence of trajectories and find synchronization it is required that $-\Lambda$ be positive definite, to this end, it is considered that $a_{i, i}=-\operatorname{deg}\left\{x_{i}\right\}=-d$; therefore, from Sylvester's criterion and for positive definiteness of $-\Lambda$

$$
\begin{gathered}
k d>0 \\
c k d>\left(\frac{1}{2}-\frac{\beta}{2} \cos \left(x_{1}\right)\right)^{2}
\end{gathered}
$$

Therefore, the contraction region for the virtual system can be defined as follows:

$$
\mathbf{C}=\left\{x \in \mathbb{R}^{2}: k>\frac{\left(1 / 2-(\beta / 2) \cos \left(x_{1}\right)\right)^{2}}{c d}, x_{2} \in \mathbb{R}\right\} .
$$

As can be seen the condition lies in the choice of the coupling constant which is a parameter that can be arbitrarily chosen provided that satisfies the corresponding condition.

Note that when $\cos \left(x_{1}\right)=-1$, we have the maximum value and, therefore, it is sufficient to choose

$$
k>\frac{(1 / 2+\beta / 2)^{2}}{c d}
$$

Therefore, with this value for the network coupling, the convergence of the trajectories of the systems is ensured and hence its synchronization. The parameters used for the systems were 


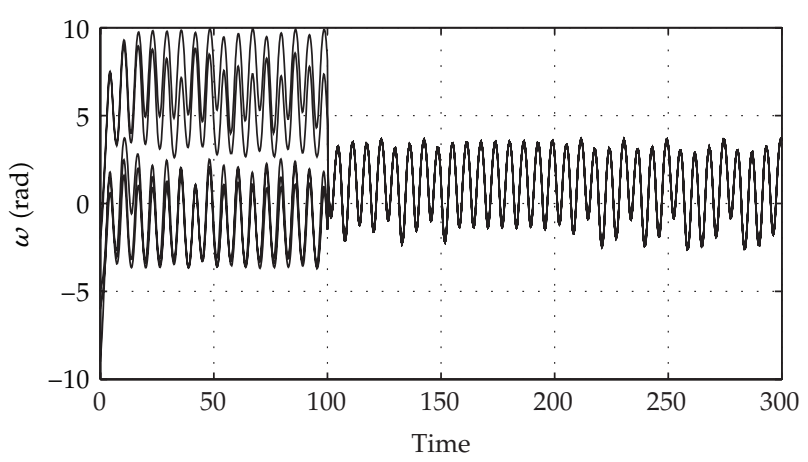

(a)

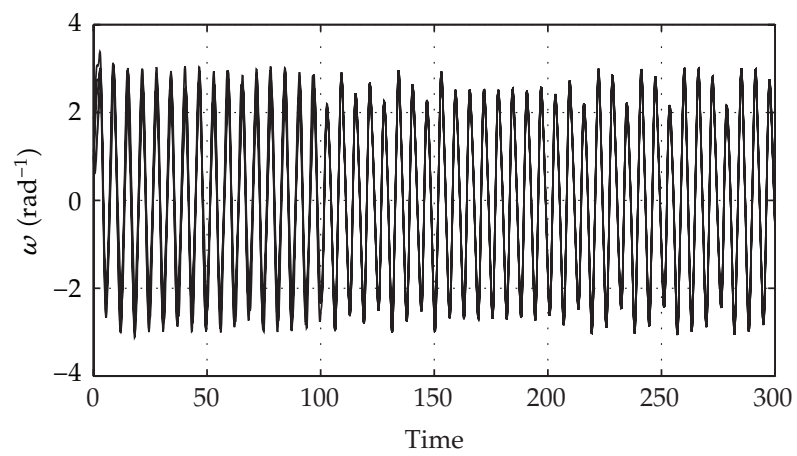

(b)

Figure 2: Evolution of the states of network systems, $\delta_{i}$ represents the angular positions of the generators, and $\omega_{i}$ the angular velocity.

chosen all equal, that in order to find the virtual system directly, the values are $h_{i}=2.45$; $\beta_{i}=1 ; c_{i}=0.5 ; \omega_{i}=1$ and the smallest degree of the network is $d=2$; therefore it is sufficient to choose $k>1$. Synchronization between the systems of the network is obtained for a value of $k=2$, that is, this value satisfies the contraction region and, therefore, the trajectories of the systems converge to those of the virtual system.

Figure 2 shows the synchronization of the systems where the generators are connected at $t=100$ and thereafter are synchronized; it should be mentioned that only angular position is considered as interconnection variable and even with this feature, position and velocity synchronization is achieved. Note that synchronization is achieved in a manifold which is not given by none of the individual systems, this manifold of synchronization is given by the interaction between systems and the network topology. Figure 3 shows the errors relative to the system (2.1), and it can be seen that errors converge to zero which implies the existence of synchronization; even though only the angular position of the generators are transmitted, also remember that a controller is not designed to achieve synchronization between systems. Thus, with this technique of analysis, an interval for the coupling parameter can be determined and with this the trajectories of generators are synchronized with each other without the use of a Lyapunov function or the calculation of Lyapunov's exponents to determine synchrony in the network; moreover, the result provides information about the robustness of the synchronization, even under the consideration of equal parameter values. 


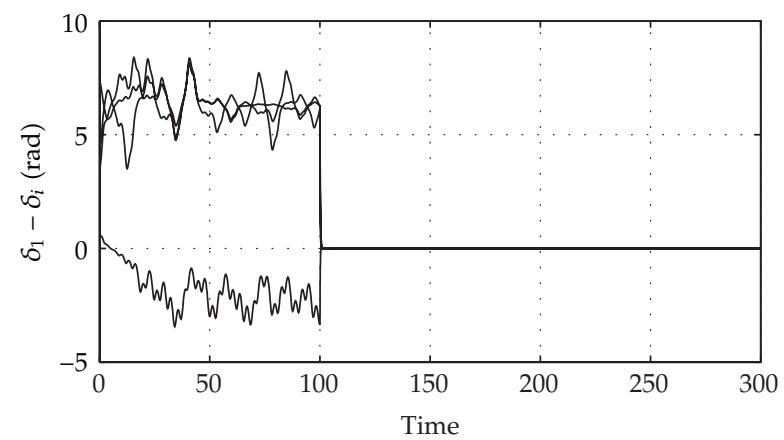

(a)

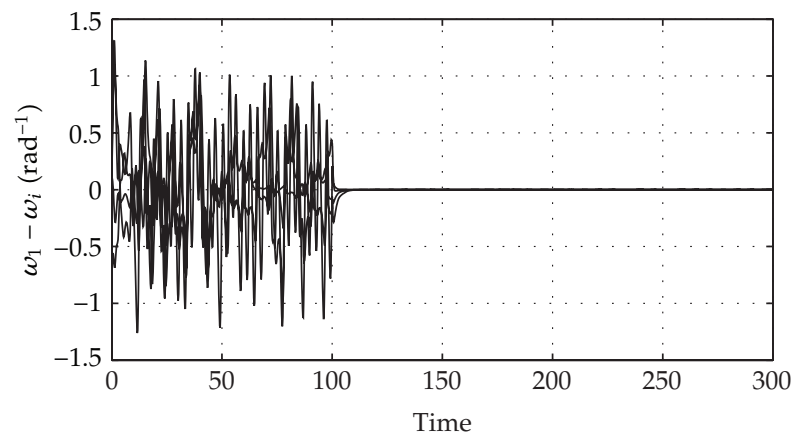

(b)

Figure 3: Position and velocity errors relative to the generator (2.1).

\section{Conclusions}

In this paper, an alternative way to carry out network synchronization of a class of power systems was presented. The chosen models consider that interact with an infinite bus. It is considered that the generators are connected in linear way through the angular position and then achieve synchronization of all generators of the network in both, position and velocity, even when the system output is only the angular position. It was also stated that the contraction theory is a good tool to study the synchronization of networks of systems. An important point is that the result is robust even when the analysis is for systems with equal parameters; moreover, the virtual system can be considered as a nominal plant, and parameter variations can be considered in the systems and the synchronization condition holds from the contraction region. This result can be extended to a larger network and also to the case when the generators are interconnected through the generated power, and this can be considered as the output of each system. Therefore, the contraction analysis can be used as an alternative technique to the Master Stability Function in the synchronization of networks of systems whith complex behavior.

\section{Acknoledgment}

This work was supported by PROMEP under the project 103.5/11/1039. 


\section{References}

[1] R. Albert and A.-L. Barabási, "Statistical mechanics of complex networks," Reviews of Modern Physics, vol. 74, no. 1, pp. 47-97, 2002.

[2] X. F. Wang and G. Chen, "Complex networks: small-world, scale-free and beyond," IEEE Circuits and Systems Magazine, vol. 3, no. 1, pp. 6-20, 2003.

[3] S. Boccaletti, V. Latora, Y. Moreno, M. Chavez, and D.-U. Hwang, "Complex networks: structure and dynamics," Physics Reports, vol. 424, no. 4-5, pp. 175-308, 2006.

[4] S. H. Strogatz, "Exploring complex networks," Nature, vol. 410, no. 6825, pp. 268-276, 2001.

[5] Z. Li and G. Chen, "Global synchronization and asymptotic stability of complex dynamical networks," IEEE Transactions on Circuits and Systems II, vol. 53, no. 1, pp. 28-33, 2006.

[6] G.-P. Jiang, W. K.-S. Tang, and G. Chen, "Global synchronization and asymptotic stability of complex dynamical networks," IEEE Transactions on Circuits and Systems, vol. 53, no. 12, pp. 2739-2745, 2006.

[7] K. Park, Y.-C. Lai, S. Gupte, and J.-W. Kim, "Synchronization in complex networks with a modular structure," Chaos, vol. 16, no. 1, Article ID 015105, 11 pages, 2006.

[8] W. Lohmiller and J.-J. E. Slotine, “On contraction analysis for non-linear systems," Automatica, vol. 34, no. 6, pp. 683-696, 1998.

[9] B. B. Sharma and I. N. Kar, "Contraction theory based adaptive synchronization of chaotic systems," Chaos, Solitons \& Fractals, vol. 41, no. 5, pp. 2437-2447, 2009.

[10] Y. S. Yang, M. L. Hung, T. L. Liao, and J. J. Yan, "Chaos synchronization in SMIB power system and its application to secure communication," Journal of Physics: Conference Series, vol. 96, no. 1, Article ID 012102, 2008.

[11] J. S. Lin, Y. S. Yang, M. L. Hung, T. L. Liao, and J. J. Yan, “Observer design for chaos synchronization of time-delayed power systems," Proceedings of World Academy of Science, Engineering and Technology, vol. 65, pp. 608-611, 2010.

[12] G. Russo and M. di Bernardo, "Contraction theory and master stability function: linking two approaches to study synchronization of complex networks," IEEE Transactions on Circuits and Systems II, vol. 56, no. 2, pp. 177-181, 2009.

[13] J.-J. E. Slotine and W. Wang, "A study of synchronization and group cooperation using partial contraction theory," in Cooperative Control, vol. 309 of Lecture Notes in Control and Information Sciences, pp. 207-228, Springer, Berlin, Germany, 2005.

[14] J. J. E. Slotine, W. Wang, and K. El Rifai, "Contraction analysis of synchronization in networks of nonlinearly coupled oscillators, in," in Proceedings from the 16th International Symposium on Mathematical Theory of Networks and Systems, Brussels, Belgium, 2004.

[15] J. Jouffroy and J. J. E. Slotine, "Methodological remarks on contraction theory," in Proceedings of the 43rd IEEE Conference on Decision and Control (CDC'04), pp. 2537-2543, December 2004.

[16] E. M. Shahverdiev, L. H. Hashimova, and N. T. Hashimova, "Chaos synchronization in some power systems," Chaos, Solitons \& Fractals, vol. 37, no. 3, pp. 827-834, 2008.

[17] Q. Lin and X. Wu, "The sufficient criteria for global synchronization of chaotic power systems under linear state-error feedback control," Nonlinear Analysis: Real World Applications, vol. 12, no. 3, pp. 15001509, 2011.

[18] F. C. Alberto, F. H. J. R. Silva, and N. G. Bretas, “Extended Lyapunov functions for detailed power system models," in Proceedings of the Power System Computation Conference, Sevilla, Spain, 2002. 


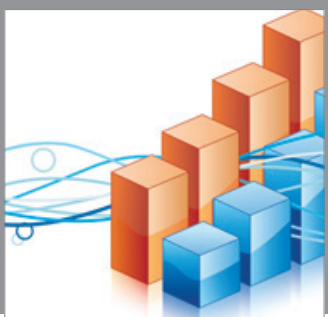

Advances in

Operations Research

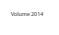

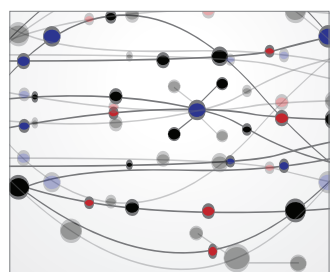

\section{The Scientific} World Journal
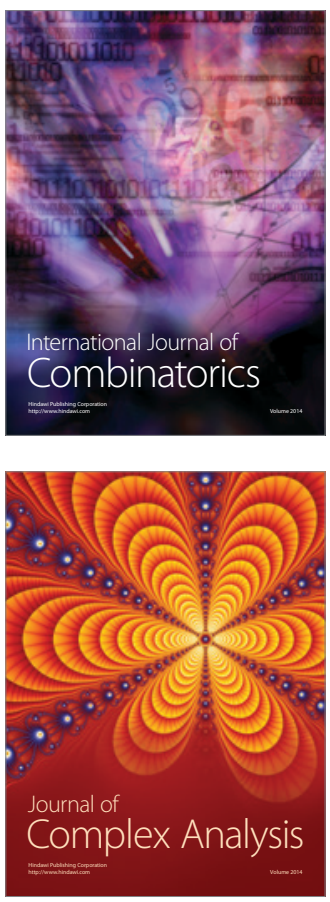

International Journal of

Mathematics and

Mathematical

Sciences
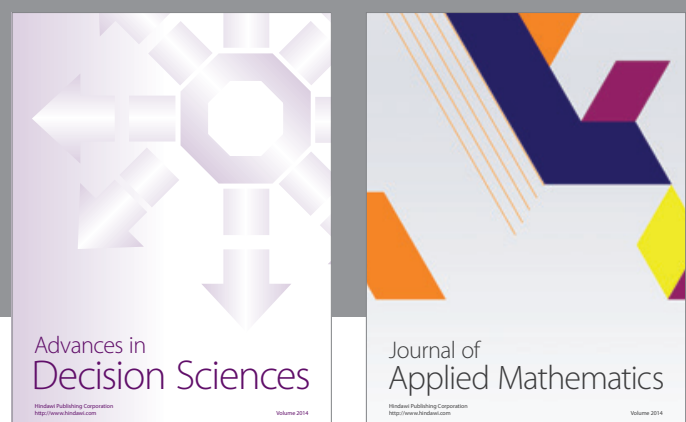

Journal of

Applied Mathematics
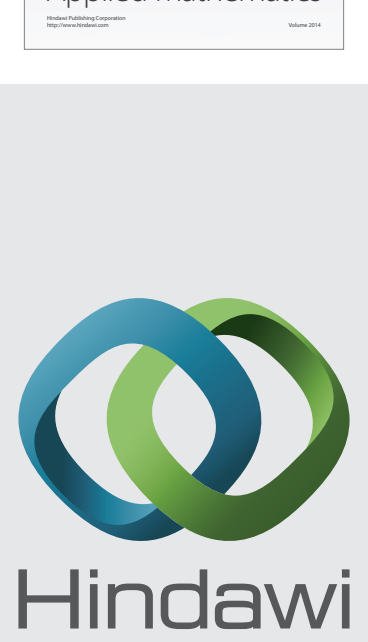

Submit your manuscripts at http://www.hindawi.com
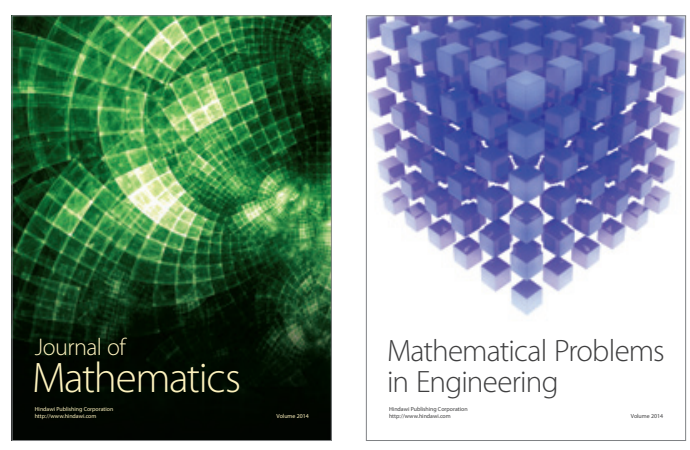

Mathematical Problems in Engineering
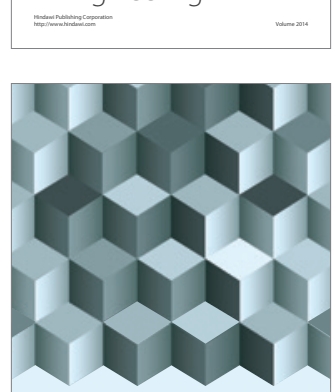

Journal of

Function Spaces
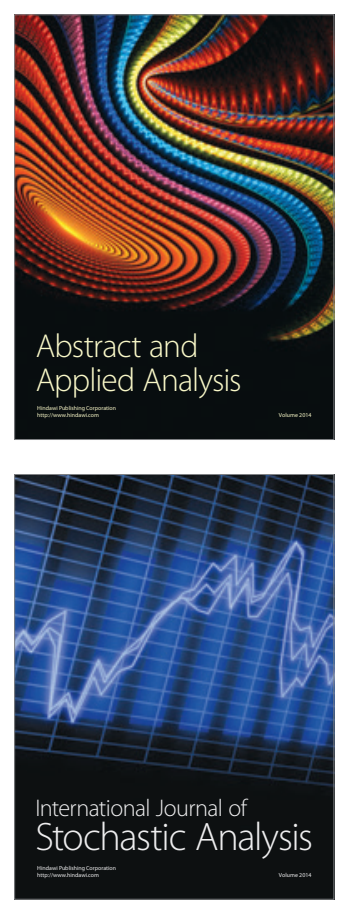

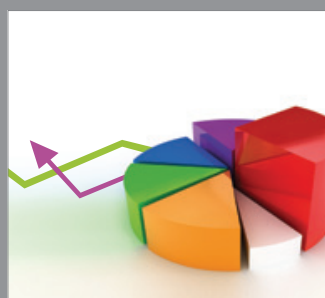

ournal of

Probability and Statistics

Promensencen
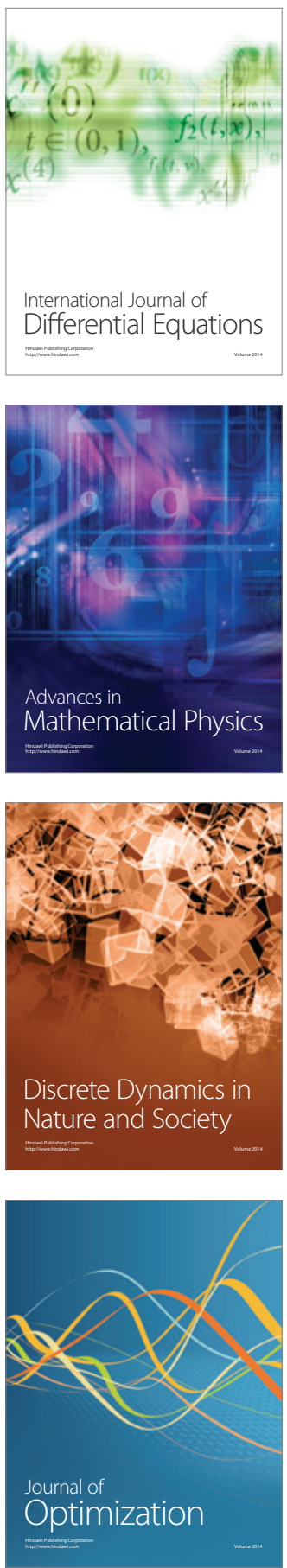